\title{
La inmunología en Antioquia: una historia con futuro. I. La creación de una Escuela
}

\author{
Luis F. García
}

\section{RESUMEN}

Mundialmente la inmunología es una de las ciencias biomédicas con mayor desarrollo en la segunda parte del siglo xx y principios del siglo xxI, además ha tenido un desarrollo muy importante en Antioquia a partir de los años sesenta del sioglo xx. En la presente reseña histórica, el autor, quien ha participado activamente durante cincuenta años como estudiante y profesor de inmunología en la Universidad de Antioquia, relata los momentos más significativos y el papel que han desempeñado algunos distinguidos profesores en el desarrollo de la investigación y la docencia de pregrado y posogrado en esta disciplina. En esta primera parte se relatan los antecedentes y los eventos que en las décadas de 1960 y 1970 permitieron establecer la inmunología como una disciplina biomédica independiente en la Universidad de Antioquia.

\section{PALABRAS CLAVE}

Antioquia; Historia; Inmunología

\section{SUMMARY}

The immunology in Antioquia: a history with future. I. The creation of a school

Immunology is one of the biomedical sciences that had undergo, worldwide, a greater development in the last part of the XX century and the beginning of the XXI century. In Antioquia, immunology also had important developments that began the 1960s. In this historical review, the author who have actively participated in these developments in the Universidad de Antioquia,

\footnotetext{
1 Profesor emérito, Grupo de Inmunología Celular e Inmunogenética, Facultad de Medicina, Sede de Investigación Universitaria, Universidad de Antioquia, Medellín, Colombia.

Correspondencia: Ifgarcia@une.net.co

Recibido: abril 12 de 2019

Aceptado: julio 4 de 2019
}

Cómo citar: García LF. La inmunología en Antioquia: una historia con futuro. I. La creación de una Escuela. latreia. 2020 Ene-Mar;33(1):84-92. D0I 10.17533/udea.iatreia.39. 
as an undergraduate and graduate student and later on as faculty member, describes the more significant local events and the role played by some distinguished faculty members in research, and in undergraduate and graduate teaching of immunology. The first part describes the historical events that occurred in the 1960 and 1970 decades that lead to the establishment of immunology as an independent biomedical.

\section{KEYWORDS}

Antioquia; History; Immunology

\section{INTRODUCCIÓN}

It is my belief that even the smallest fragments of human knowledge should be viewed in the context of all the knowledge available*

Jan KIein ${ }^{(1)}$

El presente relato pretende describir inicialmente el desarrollo histórico de la inmunología en el contexto internacional, para lograr así comprender su estado en nuestro país, en particular en Antioquia a mediados del siglo xx, específicamente en los años sesenta de este siglo junto con el rápido desarrollo de esta disciplina ocurrido posteriormente. En los tres artículos de esta serie se hará especial énfasis en la historia de tales desarrollos entre 1963 y 1995, para plantear luego, sucintamente, su estado actual $y$ formular algunas preguntas que considero relevantes sobre lo que se está haciendo en inmunología y las posibilidades futuras que avizoro desde mi perspectiva personal. Puesto que, más allá de cualquier posición pretenciosa, debo aceptar que durante las últimas cinco décadas he sido protagonista de la evolución de la inmunología en Antioquia, particularmente, en la Universidad de Antioquia. Este relato no deja de ser testimonial y en muchas de sus apartes, está escrito en primera persona. La historia de la inmunología en Antioquia fue objeto de dos publicaciones previas por Escobar ${ }^{(2,3)}$, la gran diferencia con dichas

* Traducción (Ifg): Yo creo que aún el más pequeño fragmento del conocimiento humano debe ser visto en el contexto de todo el conocimiento existente. artículos es precisamente, como ya se mencionó, que el autor del presente artículo fue uno de los actores locales de tal historia en los últimos cincuenta años, con todo lo positivo o negativo que pudiera conllevar una mirada desde adentro.

\section{CONTEXTO HISTÓRICO INTERNACIONAL}

En el período comprendido entre la segunda mitad del siglo xx y en las dos primeras décadas del presente sioglo, la inmunología ha sido una de las ciencias biomédicas que ha ostentado mayor desarrollo. La investigación experimental y clínica en inmunología ha permitido la comprensión del complejo sistema inmune, aunque todavía incompleta, y ha llegado a la vanguardia de la biomedicina al ofrecer explicaciones sobre la interacción del hospedero con los microrganismos patógenos, el reconocimiento de los autoantígenos y la tolerancia frente a los mismos, así como las consecuencias de una respuesta autoinmune. Igualmente, se ha investigado las reacciones a los alergenos, los antígenos tumorales, los trasplantes de células y tejidos y muchos otros fenómenos biológicos de importancia para la supervivencia de los individuos y las especies, incluyendo por supuesto, al Homo sapiens.

En forma simplificada la evolución histórica de la inmunología puede dividirse en diferentes eras (Tabla 1).

\section{Tabla 1. Las eras en la historia de la inmunología}

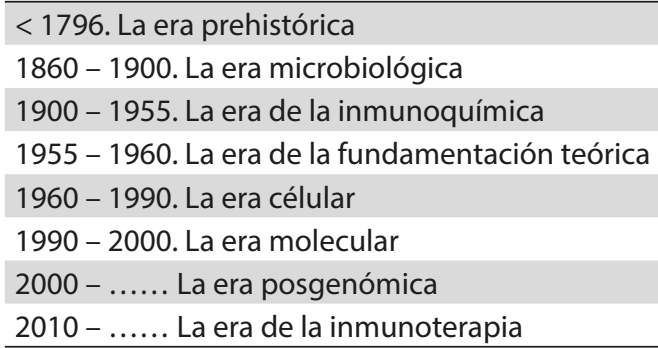

Fuente: creación propia

Desde la antigüedad se propusieron explicaciones sobre la resistencia a las pestes en aquellos individuos que, habiendo estado expuestos, sobrevivieron, para luego ser los encargados de cuidar a los enfermos 
cuando se presentaba una nueva epidemia. Pero no fue hasta 1796 que Edward Jenner (1749-1823) descubrió la vacunación contra la viruela y se dispuso de un procedimiento confiable y seguro para ser utilizado en forma masiva en la profilaxis de una de las más importantes plagas que ha sufrido la humanidad. En la segunda mitad del siglo xIX, durante la época de oro de la microbiología, Louis Pasteur (1822-1895) encontró la forma de inmunizar contra el cólera de las gallinas, el ántrax y la rabia usando microorganismos atenuados y, Emil von Boehring (1854-1917) descubrió la forma de producir sueros con actividad de antitoxinas en el caso de la difteria y del tétanos, sueros que podían ser empleados para la inmunización pasiva en la prevención y el tratamiento de estas enfermedades. Sin embaroo, el nacimiento de la inmunología como una disciplina biomédica, con su propio contexto teórico y metodológico, tuvo lugar en 1899 cuando Paul Ehrlich (1854-1915) postuló la primera teoría de la respuesta inmune y creó una terminología propia que aún se utiliza ${ }^{(4)}$. Esta, conocida como la teoría de las cadenas laterales, postula que las células tienen en sus membranas cadenas que reconocen las toxinas $y$, como resultado de este reconocimiento, dichas cadenas son liberadas a los fluidos corporales en forma de antitoxinas.

A finales del siglo xIx y principios del siglo xx se inició también la identificación de las células que participaban en la respuesta inmune, particularmente, Ilich Metchnikoff (1845-1916) demostró la capacidad de algunas células de fagocitar y destruir diferentes microorganismos. Sin embargo, el desarrollo de la biología celular en esos años era aún muy precario, comparativamente con el desarrollo de otras ciencias como la química, de tal manera que en la primera mitad del siglo xx la inmunología estuvo dominada por la comprensión de la reacción antígeno-anticuerpo en términos químicos.

Esto permitió el desarrollo de un número importante de métodos serológicos para el diagnóstico de las enfermedades infecciosas y la clasificación de los serotipos de microoroganismos. Fue solo a mediados del siglo xx, cuando los desarrollos de la biología celular y la genética permitieron postular las modernas teorías selectivas que abrieron la caja de Pandora del sistema inmune. En 1957, MacFarlane Burnet (1899-1985) postuló la teoría de la selección clonal ${ }^{(5)}$, que propone que los linfocitos son los responsables del reconocimiento de los antígenos mediante receptores, distribuidos clonalmente, presentes en sus membranas y cuya diversidad se genera durante la vida fetal, en forma independiente del antígeno. Luego en la vida extrauterina la exposición a antígenos particulares permite que las clonas de las células inmunocompetentes con receptores que reconocen estos antígenos se expandan y diferencien en las células efectoras y productoras de anticuerpos específicos. Esta teoría permitió las aproximaciones experimentales que llevaron a la identificación de las poblaciones de linfocitos $\mathrm{T}$ y $\mathrm{B}$, sus interacciones, su regulación, sus productos $y$ las alteraciones que se presentan en muchas enfermedades. A finales del siglo xx los avances de la biología molecular permitieron entender los mecanismos de expresión y regulación de los genes involucrados en la diferenciación $y$ función de las células inmunes y las señales que median estos fenómenos. En el presente siglo, la inmunología en la era posgenómica, avanza en la elaboración de modelos que permiten la comprensión sistémica de las respuestas inmunes normales y patológicas, además la industria farmacéutica y biotecnológica han lanzado al mercado una gran cantidad de moléculas inmunológicas con actividad terapéutica en múltiples ramas de la medicina ${ }^{(6-8)}$.

En Colombia durante la primera parte del sioglo xx la inmunología estuvo al servicio de las enfermedades infecciosas, cuando se hicieron esfuerzos importantes para la producción de vacunas, como la antivariólica producida en el Parque de las Vacunas gracias al esfuerzo de pioneros como Jorge Lleras Parra $(1874-1945)^{(9)}$, o la producción en el Laboratorio Samper Martínez (hoy el Instituto Nacional de Salud) de vacunas para la fiebre amarilla y para la tuberculosis con el Bacilo de Calmette y Guerin (BCG). (Bacilo de Calmette y Guerin).

Asimismo, se implementaron técnicas serológicas, como el VDRL, para el diaơnóstico de la sífilis y la llamada "seroaglutinación para febriles" para el diagnóstico de infecciones bacterianas entéricas como las salmonelosis, las cuales fueron utilizadas, inclusive, con fines laborales. Igualmente se usaron otras técnicas de inmunohematología en los bancos de sangre. ${ }^{* *}$ En las escuelas de medicina se incluía, dentro del curso de microbiología, una o dos clases para explicar las técnicas serológicas existentes, a saber: la aglutinación, la precipitación y la fijación de complemento, pero no se impartía enseñanza

\footnotetext{
* Entrevistas con los profesores Marcos Restrepo, Fabiola Montoya de Restrepo, Medellín, 4 de abril de 2018, Medellín.

Entrevista con el profesor William Rojas, Medellín, 18 de abril de 2018, Medellín.
} 
adicional sobre el funcionamiento del sistema inmune y sus componentes, ni se mencionaban las posibles patologías asociadas con la respuesta inmune.

\section{LA CREACIÓN DE UNA ESCUELA}

En la Facultad de Medicina de la Universidad de Antioquia la inmunología se inició en 1963 con la creación del Laboratorio de Inmunología, adscrito al Departamento de Microbiología y Parasitología a caroo de Marcos Restrepo (Figura 1), recién vinculado como docente luego de su grado de médico. Bajo su dirección el Laboratorio de Inmunología abrió un espacio académico e investigativo para esta disciplina que aún hoy continúa en expansión.

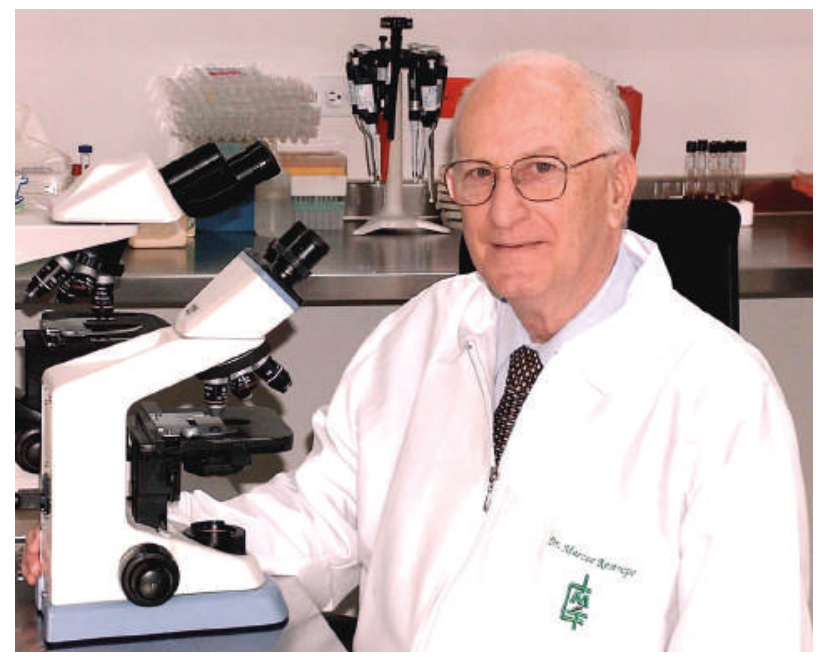

Figura 1. Marcos Restrepo, fundador en 1963 del Laboratorio de Inmunología del Departamento de Microbiología y Parasitología de la Facultad de Medicina de la Universidad de Antioquia. Dirigió el Laboratorio hasta 1976 cuando se retiró de la Universidad. (Fotografía gentilmente proporcionada por Marcos Restrepo)

No puedo continuar sin mostrar como un testimonio de este proceso las dedicatorias escritas en un ejemplar de la tercera edición del libro Experimental Immunochemistry de Elvin Kabat y Manfred Meyer ${ }^{(10)}$, el cual fue un clásico en los años cuarenta y cincuenta del siglo xx. Este ejemplar fue adquiriđo por Ángela Restrepo en 1960 cuando realizaba sus estudios de maestría en la Universidad de Tulane en New Orleans (Estados Unidos), pero el 8 de mayo de 1962 se lo obsequió a Marcos Restrepo, con la dedicatoria "Para nuestro futuro 'inmunólogo' con muchos buenos deseos!". Alounos años después, el 25 de noviembre de 1970, Marcos Restrepo me lo obsequió con la dedicatoria "Para nuestro futuro 'inmunólogo" (Figura 2). Aún conservo este ejemplar y lo consulto con frecuencia para mis seminarios sobre la historia de la inmunología.

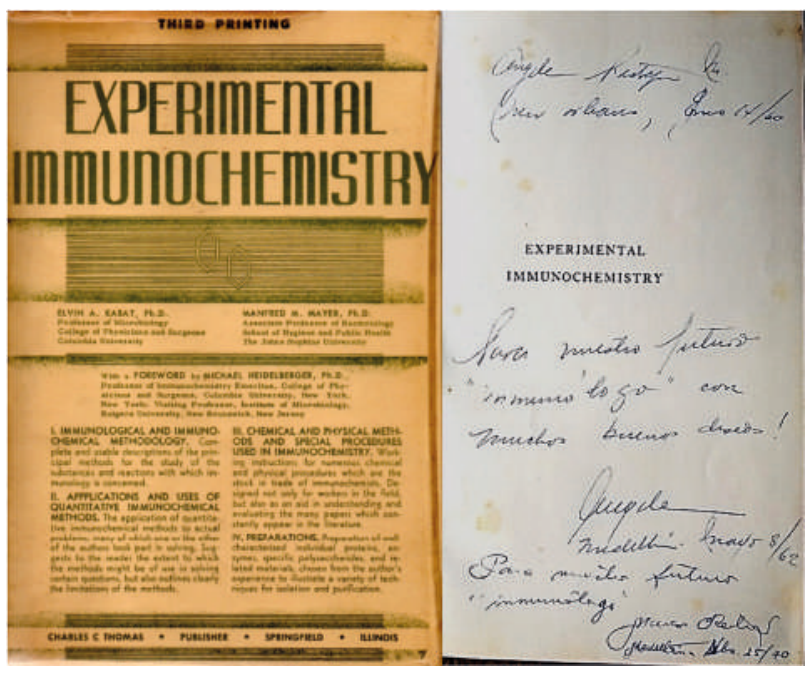

Figura 2. El nacimiento de una escuela. El libro clásico de Elvin A. Kabat y Manfred M. Mayer Experimental Immunochemistry. 3ra. ed, 1958, fue adquirido por Ángela Restrepo en New Orleans durante sus estudios de Maestría en Tulane University. El 8 de mayo de 1962 se lo obsequia a Marcos Restrepo, quien el 25 de noviembre de 1970 lo obsequia al autor. Fuente: creación propia

\section{EL LABORATORIO DE INMUNOLOGÍA}

El interés principal de Marcos Restrepo era, y continúa siéndolo, el estudio de las enfermedades tropicales y su mayor fortaleza técnica, al igual que de la bacterióloga Fabiola Montoya de Restrepo, también vinculada al Laboratorio de Inmunología en esos años, eran las reacciones serológicas. Esta colaboración se reflejó en la implementación de nuevos procedimientos serológicos como la fijación de complemento, la hemaglutinación pasiva y la inmunodifusión en dos dimensiones de Ouchterlony, para el estudio de 
enfermedades como la amibiasis ${ }^{(11)}$, la enfermedad de Chagas, entre otras. Con la adquisición en 1967 de dos microscopios marca Leitz de inmunofluorescencia y condensador de contraste de fase, uno para el Departamento de Microbiología y Parasitología y otro para el Departamento de Patología, además de la capacitación adquirida en los cursos dictados por Jovan Gutic del Instituto de Medicina Tropical de la Universidad Central de Venezuela, fue posible implementar diversas técnicas de inmunofluorescencia para el diagnóstico de la sífilis ${ }^{(12,13)}$ y la toxoplasmosis, entre otras.

Una contribución muy importante del profesor Restrepo fue expandir la inmunología a áreas diferentes a las enfermedades infecciosas y parasitarias mediante la colaboración de especialistas de otras disciplinas y, la vinculación de personal técnico dedicado a estos nuevos espacios de trabajo, se introduce de esta manera la inmunología moderna a las siguientes áreas:

\begin{abstract}
Alergología: con la participación de los internistas Jorge Hincapié y Alberto Robledo, el pediatra Ricardo Garcés y el propio Marcos Restrepo, se inició una consulta externa de alergias que fue respaldada por la preparación de alergenos para el polvo casero y algunos hongos por la bacterióloga María Elena Sánchez, que además realizaba las pruebas intradérmicas y de parche con una batería de alergenos comerciales y posteriormente aplicaba la terapia de hiposensibilización. A esta consulta se vinculó, posteriormente, Fernando Vío del Río, médico aleróólogo chileno que vivió varios años en Medellín, luego Fernando Montoya, Luis Rafael Caraballo y mi persona, los tres en condición de médicos y estudiantes de maestría.
\end{abstract}

Autoinmunidad: con la participación de los reumatólogos Javier Molina y Carlos Agudelo, junto con el apoyo técnico de la bacterióloga María Inés Uribe se implementaron procedimientos para el estudio de enfermedades como el lupus eritematoso sistémico y la artritis reumatoidea. Entre estos procedimientos estaban la detección de las células LE, la determinación de anticuerpos antinucleares por inmunofluorescencia, la cuantificación del complemento sérico por la técnica del $\mathrm{CH}_{50}$ y la de las fracciones $\mathrm{C} 2, \mathrm{C} 3$ y $\mathrm{C} 4$ por inmunodifusión radial, además de la reducción del nitroazul de tetrazolio (NBT) por polimorfonucleares neutrófilos ${ }^{(14-16)}$.
Trasplantes: con el liderazgo del nefrólogo Jaime Borrero y del cirujano Álvaro Velázquez se creó el Grupo de Trasplantes de la Universidad de Antioquia y del Hospital Universitario San Vicente de Paúl. Este grupo realizó en agosto de 1973 el primer trasplante renal exitoso en Colombia, en el que se utilizó un donante vivo emparentado con el enfermo ${ }^{(17)}$. Al Grupo de Trasplantes se vinculó Marcos Restrepo como inmunólogo y fue él quien llevó las muestras del receptor ४ del donante de este primer trasplante, para hacer el estudio de HLA y de anticuerpos citotóxicos en el laboratorio de Paul Terasaki, en la Universidad de California en Los Ángeles (UCLA). Entre 1973 y 1977 los estudios de anticuerpos citotóxicos previos al trasplante renal continuaron siendo practicados en el Laboratorio de Inmunología por la bacterióloga Fabiola Montoya de Restrepo.

Neoplasias del sistema inmune: con la implementación de las técnicas de inmunoelectroforesis e inmunodifusión radial a principios de los años setenta, fue posible identificar y cuantificar las inmunoglobulinas, lo cual permitió colaborar con los hematólogos Alberto Restrepo y Leonardo Arango para hacer la primera clasificación inmunológica de mieloma múltiple ${ }^{(18)}$, describir el primer caso de macroglobulinemia de Waldenstrom en el país ${ }^{(19)}$, y estudiar los niveles de inmunoglobulinas en diferentes tipos de leucemias ${ }^{(20)}$.

Inmunodeficiencias: la existencia del Laboratorio de Inmunología permitió el estudio de pacientes con inmunodeficiencias ${ }^{(21.22)}$, un área que luego alcanzaría un gran desarrollo que se mantiene hasta el momento, como se describirá en la segunda parte de este trabajo.

Estudio de células del sistema inmune: a principios de los años setenta también se implementaron las primeras técnicas para la identificación de linfocitos $\mathrm{T}$ y B, particularmente, las denominadas rosetas E, EA y EAC y la de determinación de inmunoglobulinas de membrana para linfocitos B. Estas técnicas las apliqué personalmente en tuberculosis, bajo la dirección del profesor Restrepo para mi tesis de maestría ${ }^{(23)}$.

La colaboración del Laboratorio de Inmunología con otras especialidades fue tan estrecha que en julio de 1973 el Consejo Académico de la Facultad de Medicina solicitó, al entonces rector de la Universidad de Antioquia Luis Fernando Duque, la creación de la Sección 
de Inmunología, Reumatología y Aleroias como una "unión entre los departamentos de Medicina Interna y de Microbiología y Parasitología" y, la designación de Marcos Restrepo como su director, quien ocupó el cargo hasta 1976, cuando se retiró de la Universidad para luego ser director del Laboratorio Departamental, más tarde se convierte en el director del Instituto Colombiano de Medicina Tropical, hoy dependencia de la Universidad CES. Según su propio testimonio, después de su retiro de la Universidad de Antioquia, el profesor Restrepo no hizo más investigación en inmunología, pero sí dictó cursos de esa signatura en la Universidad Pontificia Bolivariana y la Universidad CES.

\section{LOS PRIMEROS CURSOS DE INMUNOLOGÍA}

En 1966 se dictó en la Facultad de Medicina el primer curso de inmunología ${ }^{(3)}$, por Roger Bolaños profesor de la Universidad de Costa Rica, quien fue compañero de Ángela Restrepo en el doctorado en la Universidad de Tulane. Sin embargo, el curso que turo un impacto decisivo en el desarrollo de la inmunología en nuestra universidad fue dictado en noviembre de 1970 por el profesor Quentin N. Myrvik de Wake Forest University, Winston-Salem, NC (Estados Unidos). Este curso fue organizado por Ángela Restrepo dentro del programa Latin American Professorship de la American Society for Microbiology (ASM) (Figura 3), y asistieron los profesores Ángela Restrepo, Marcos Restrepo, Federico Díaz y Eduardo Leiderman, algunos residentes de medicina interna y de pediatría, destacándose la residente de pediatría Diana García de Olarte; estudiantes de medicina y de la Maestría en Microbiología y Parasitología, particularmente, Fernando Montoya y mi persona, así como Luis Rafael Caraballo, en ese entonces estudiante de medicina en la Universidad de Cartagena.

\section{LAS MAESTRÍAS EN CIENCIAS BÁSICAS}

En 1969 se iniciaron en la Facultad de Medicina varios programas de maestría en ciencias básicas biomédicas que buscaban la formación de docentes e investigadores en morfología, bioquímica, fisiología, farmacología, parasitología y microbiología. Dentro de esta última, se abrió la posibilidad de hacer énfasis en inmunología, por lo que en 1975 tuve el honor de ser el primer graduado en esta conđición con un trabajo titulado Células

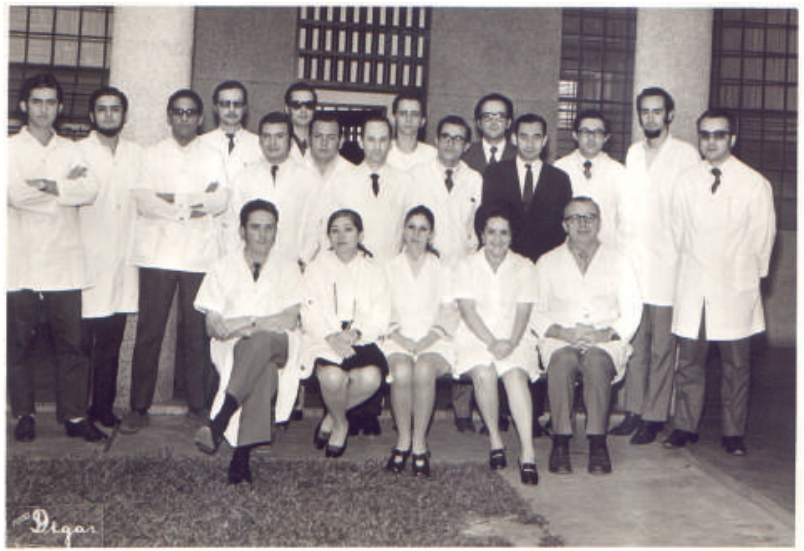

Figura 3. Curso de Inmunología Avanzada dictado por el profesor Quentin N. Myrvik.t (Wake Forest University, Winston-Salem, NC, U.S.A.), auspiciado por la American Society for Microbiology (ASM), Latin American Professorship, Universidad de Antioquia, Medellín, noviembre 9-27, 1970. Fuente: creación propia

formadoras de rosetas en pacientes con tuberculosis. Con este énfasis recibieron también el título de magíster en Microbiología Médica, algunos años más tarde, los médicos Fernando Montoya, Luis Rafael Caraballo y la bacterióloga Luz Aristizábal, los tres bajo mi đirección como detallaré más adelante. Estos programas fueron clausurados en 1982 por la Universidad, pero permitieron formar un número importante de profesores e investigadores de ciencias básicas biomédicas para la Universidad de Antioquia y otras universidades como la Universidad Industrial de Santander, la Universidad de Cartagena y la Universidad del Cauca.

\section{LAS PRIMERAS PUBLICACIONES INTERNACIONALES}

Paradójicamente las primeras publicaciones internacionales relacionadas con la inmunología no se

† De izquierda a derecha. Fila posterior: Nicolás Pulido, Jaime Carmona, Luis Rafael Caraballo, Fernando Montoya, Luis Fernando Velásquez, Jaime Moreno, Emilson Guzmán, Marcos Restrepo, Roberto Giraldo, Wilfredo Caicedo, Óscar Villegas, Sr. Carvajal, Eduardo Leiderman, Luis F. García, Óscar Ruiz. Primera Fila: Federico Díaz, Diana García de Olarte, Ligia Pérez, Ángela Restrepo, Quentin N. Myvik. 
generaron en el Laboratorio de Inmunología sino en otras dependencias. Una de ellas fue llevada a cabo en el Laboratorio de Nutrición dirigido por el médico internista Hernán Vélez, que logró en los primeros años de la década del setenta una fructífera colaboración con el profesor de la Boston University, Joseph Vitale, quien le permitió hacer estudios clásicos en diferentes temas relacionados con la nutrición y dotar el mejor laboratorio de la Facultad en su momento. producto de esa colaboración surge el artículo de Munson et al., ${ }^{(24)}$, el cual contó con la coautoría de Hernán Vélez y del pediatra Darío Franco. En este artículo se estudiaron los niveles séricos de IogG, IogM e IogA, la fagocitosis por polimorfonucleares neutrófilos y la respuesta tuberculínica en niños $y$ adultos con desnutrición calórico-proteica.

Otras publicaciones dignas de mencionar fueron realizadas en el Laboratorio de Micología del Departamento de Microbiología y Parasitología por Ángela Restrepo como parte de su productiva línea de investigación en paracoccidioidomicosis. En la primera describió la estandarización de la inmunodifusión de Ouchterlony para el diagnóstico de esta micosis ${ }^{(25)}$, y en la segunda publicación, en colaboración con la bacterióloga Herta Vélez ${ }^{(26)}$, estudió la capacidad de las células polimorfonucleares humanas para fagocitar levaduras de Paracoccidioides brasiliensis y Rodothorula sp., en presencia de suero autólogo o suero $\mathrm{AB}$. La importancia de esta investiogación residió en que, por primera vez, se estudiaba in vitro la capacidad funcional de células del sistema inmune.

Una publicación más para mencionar, realizada con Antonio Carlos Jaramillo, compañero de maestría, se llevó a cabo bajo la dirección de Marcos Restrepo en el Laboratorio de Inmunología ${ }^{(27)}$. En este estudio se hizo una comparación antigénica de tres cepas de Toxoplasma oondii y una de Besnoitia jellosonii, resultado de una investigación esencialmente básica que incluía un parásito no humano (B. jellisonii), relacionado filogenéticamente con el patógeno humano $T$. gondii.

\section{ENTRENAMIENTO EN ESTADOS UNIDOS}

En 1970 durante el curso de Inmunología Avanzada dictado por el profesor Quentin N. Myrvik se gestionó por parte de Ángela Restrepo y Marcos Restrepo, que una vez terminara mi formación como médico y magíster en Microbiología Médica en la Universidad de Antioquia, viajaría a Estados Unidos para ingresar al programa de Ph. D. en el Department of Microbiology and Immunology de la Wake Forest University, cuyo jefe era el profesor Myrvik. Efectivamente, en diciembre de 1974 viajé a Estados Unidos con un nombramiento como docente ad honorem de la Universidad de Antioquia e inicié mis estudios doctorales en tal universidad tomando los cursos requeridos para ese programa para dar inicio con el proyecto de tesis. En este proyecto usé un modelo experimental de tuberculosis en conejos, inoculados por vía intravenosa o intratraqueal con Mycobacterium bovis (BCG), para luego determinar en los lavados pulmonares y en los sobrenadantes de los cultivos de los linfocitos obtenidos en estos lavados, de células esplénicas y de nódulos linfáticos, la presencia del factor inhibidor de la mioración de macrófagos (MIF) y de un hipotético factor aoglutinante de los macrófagos (MAgF) ${ }^{(28)}$. En condiciones económicas difíciles, se termina con éxito el primer semestre. En consecuencia, se plantea al tutor dicha situación, quien propone que en mi condición de médico graduado podía suspender el programa de doctorado y ser contratado como "Posdoctoral Fellow" y en ese caso los recursos destinados a la matrícula se me entregarían como salario de "posdoc", dicha propuesta estaba condicionada a tomar los cursos de inmunología, que debería haber tomado en el programa de $\mathrm{Ph}$. D., como cursos de servicio. La experiencia de esos dos años de entrenamiento en inmunología fue invaluable, tanto desde el punto de vista de mi formación científica como personal. Debo hacer expreso reconocimiento al profesor Myrvik y al profesor David Groves. De este último aprendí el método de enseñar diseñando hipotéticos proyectos de investigación basados en los artículos que se entregan a los estudiantes, metodología que he empleado en todos mis años como docente y, que me acercó a la bioestadística como herramienta fundamental para el trabajo experimental.

Durante el tiempo que esture en ese entrenamiento manture contacto permanente con mis antiguos profesores en Colombia y, la decisión de suspender el programa de $\mathrm{Ph}$. D., fue consultada con ellos. Igualmente, en esos años Ángela Restrepo ya participaba en el Comité de Colciencias que asiognaba recursos para investigación y, por invitación suya se propone a dicha instancia un proyecto que planeaba replicar 
el modelo de granulomas pulmonares en conejos inoculados con Paracoccidioides brasiliensis en lugar de BCG, este proyecto fue aprobado por esa institución. Lo anterior permitió, al momento de regresar a CoIombia, contar con algunos recursos económicos para dar continuidad al trabajo que se venía realizando.

\section{CONFLICTO DE INTERESES}

Ninguno por declarar.

\section{REFERENCIAS BIBLIOGRÁFICAS}

1. Klein J. Natural History of the Major Histocompatibility Complex. New York: John Wiley \& Sons; 1986.

2. Escobar-Gónima C. La inmunología en Antioquia: primera parte. Iatreia. 2000;13:179-84.

3. Escobar-Gónima, C. La inmunología en Antioquia. Segunda Parte. Iatreia 2001;14:211-5.

4. Ehrlich P. The Croonian Lecture.-On Immunity with special reference to cell life. Proc Roy Soc London. 1997;66:424-48.

5. Burnet FM. A Modification of Jerne's theory of antibody production using the concept of clonal selection. Austr J Sci. 1957;20:67-9.

6. Smith KA. Towards a molecular understanding of adaptive immunity: a chronology, part I. Front Immunol. 2012 Dec;3:369. DOI 10.3389/fimmu.2012.00369.

7. Smith KA. Towards a molecular understanding of adaptive immunity: a chronology, part II. Front Immunol. 2012;3:364. DOI 10.3389/fimmu.2012.00364.

8. Smith KA. Towards a molecular understanding of adaptive immunity: a chronology, part III. Front Immunol. 2014 Feb;5:29. DOI 10.3389/fimmu.2014.00029.

9. Salamanca Uribe J. Jorge Lleras Parra y la producción de la vacuna antivariólica en Colombia. Rev acad colomb cienc. 2004;28(109):545-54.

10. Kabat EA, Mayer MM. Experimental Immunochemistry. 3 ed. Springfield: Charles Thomas; 1958.

11. Restrepo M, de Restrepo F, Botero D. Reacciones serológicas en pacientes con amibiasis. Acta Med Colombiana 1976;4(1):223-8.

12. Restrepo A, Restrepo M, Ochoa N, Giraldo R. Comparación de la técnica de anticuerpos fluo- rescentes absorbidos (FTA-ABS) con el VDRL y la fijación del complemento (Reiter) en el serodiagnóstico de la sífilis. Antioquia Med. 1970;20:147-56.

13. Peña R, Restrepo M. Inmunoglobilina $M$ en el diagnóstico de sífilis congénita. Antioquia Med. 1976; 26:71-86.

14. Restrepo M, Bustamante A. Complemento sérico en individuos normales. Antioquia Med. 1976;22:617-24.

15. Restrepo M, Molina J, Aristizábal L, Agudelo C. Niveles de complemento sérico total en varias entidades clínicas. Antioquia Med. 1974;24:35-46

16. Restrepo M, Agudelo C, Molina J, Aristizábal L. Aplicación diagnóstica del Nitroazul de tretrazolium. Acta Med Colombiana. 1977;2:159-65.

17. Borrero RJ. Transplantes renales. Medellín: Bedout; 1977.

18. Arango L, García LF, Restrepo A. Mieloma de células plasmáticas: estudio de 44 casos. Antioquia Médica. 1975;25:569-89.

19. Arango L, Hidrón H, García LF. Macroglobulinemia de Waldenstrom. Antioquia Méd. 1976;26:253-7.

20. Restrepo A, Restrepo M, Maya LM, Pérez S. Valores de inmunoglobulinas séricas en leucemias y sus variaciones con la quimioterapia. Acta Med Colombiana 1976;1:121-7.

21. Restrepo J, Restrepo A, Bojanini E, Bustamante J, Restrepo M, Vélez H. Agamaglobulinemia adquirida primaria: presentación de un caso. Antioquia Med. 1965; 15:53-66.

22. Restrepo A, Restrepo M, Maya LM. Neutropenia crónica familiar benigna. Acta Med Colombiana. 1977;2:11-8

23. García LF. Linfocitos formadores de Rosetas en pacientes tuberculosos. [Tesis Maestría en Microbiología Médica]. Medellín: Universidad de Antioquia; 1974.

24. Munson D, Franco D, Arbeter A, Velez H, Vitale JJ. Serum levels of immunoglobulins, cell-mediated immunity, and phagocytosis in protein-calorie malnutrition. Am J Clin Nutrit. 1974;27:625-8.

25. Restrepo A. La prueba de la inmunodifusión en el diagnóstico de la Paracoccidioidomicosis. Sabouraudia, 1966;4:223-30. 
26. Restrepo MA, Vélez AH. Efectos de la fagocitosis in vitro sobre el Paracoccidioides brasiliensis. Sabourodia. 1975;13:10-21.

27. Jaramillo AC, Restrepo M, García LF. Comparación antigénica entre tres cepas de Toxoplasma gondii y una de Besnoitia jellisonii. Rev Latinoam Microbiol. 1976;18:93-6.

28. García-Moreno LF, Myrvik QN. Macrophage-Agglutinating Factor produced in vitro by BCG-sensitized lymphocytes. Infect Immunity.1977;17:613-20. 